\title{
Propagation of Singularities in the Solutions to the Boltzmann Equation near Equilibrium
}

\author{
Renjun Duan ${ }^{\mathrm{a}}$, Meng-Rong Li ${ }^{\mathrm{b}}$, Tong Yang ${ }^{\mathrm{a}}$

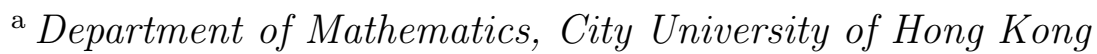 \\ Kowloon, Hong Kong, P.R. China \\ b Department of Mathematical Sciences, National Chengchi University \\ Taipei, Taiwan
}

$2007-10-18$

\begin{abstract}
This paper is about the propagation of the singularities in the solutions to the Cauchy problem of the spatially inhomogeneous Boltzmann equation with angular cutoff assumption. It is motivated by the work of Boudin-Desvillettes on the propagation of singularities in solutions near vacuum. It shows that for the solution near a global Maxwellian, singularities in the initial data propagate like the free transportation. Precisely, the solution is the sum of two parts in which one keeps the singularities of the initial data and the other one is regular with locally bounded derivatives of fractional order in some Sobolev space. In addition, the dependence of the regularity on the cross-section is also given.
\end{abstract}

\section{Introduction}

Consider the Cauchy problem of the Boltzmann equation

$$
\partial_{t} f+\xi \cdot \nabla_{x} f=Q(f, f)
$$

with initial data

$$
f(0, x, \xi)=f_{0}(x, \xi) .
$$

Here $f=f(t, x, \xi)$ is a non-negative function standing for the number density of gas particles with position $x=\left(x_{1}, x_{2}, x_{3}\right) \in \mathbb{R}^{3}$ and velocity $\xi=\left(\xi_{1}, \xi_{2}, \xi_{3}\right) \in \mathbb{R}^{3}$ at time $t>0$. $Q$ is the bilinear collision operator defined by

$$
\begin{gathered}
Q(f, g)=Q^{+}(f, g)-f L g, \\
Q^{+}(f, g)=\iint_{\mathbb{R}^{3} \times S^{2}} f\left(t, x, \xi^{\prime}\right) g\left(t, x, \xi_{*}^{\prime}\right) B\left(\xi-\xi_{*}, \frac{\xi-\xi_{*}}{\left|\xi-\xi_{*}\right|} \cdot \omega\right) d \xi_{*} d \omega, \\
L g=\iint_{\mathbb{R}^{3} \times S^{2}} g\left(t, x, \xi_{*}\right) B\left(\xi-\xi_{*}, \frac{\xi-\xi_{*}}{\left|\xi-\xi_{*}\right|} \cdot \omega\right) d \xi_{*} d \omega,
\end{gathered}
$$


where the relation between the post-collision velocity pair $\left(\xi^{\prime}, \xi_{*}^{\prime}\right)$ of two particles with the pre-collision velocity pair $\left(\xi, \xi_{*}\right)$ is given by

$$
\xi^{\prime}=\xi-\left(\xi-\xi_{*}\right) \cdot \omega \omega, \quad \xi_{*}^{\prime}=\xi_{*}+\left(\xi-\xi_{*}\right) \cdot \omega \omega, \omega \in S^{2} .
$$

Here, $B(\cdot, \cdot)$ depending only on $\left|\xi-\xi_{*}\right|$ and $\left(\xi-\xi_{*}\right) \cdot \omega /\left|\xi-\xi_{*}\right|$ is called the cross section characterizing the collision of gas particles for various interaction potentials. As usual, set

$$
A(z)=\int_{S^{2}} B\left(z, \frac{z}{|z|} \cdot \omega\right) d \omega, \quad z \in \mathbb{R}^{3} .
$$

Then $L g$ can be written as

$$
L g=A *_{\xi} g=\int_{\mathbb{R}^{3}} A\left(\xi-\xi_{*}\right) g\left(t, x, \xi_{*}\right) d \xi_{*} .
$$

In what follows, we assume that

A1. $B(\cdot, \cdot)$ is a non-negative measurable function in the form of

$$
B(z, \cos \theta)=|z|^{\gamma} b(\cos \theta), \quad \cos \theta=\frac{z}{|z|} \cdot \omega .
$$

Here $0 \leq \gamma \leq 1$, and $b(\cdot)$ satisfies the Grad's angular cutoff assumption [15], with

$$
\int_{S^{2}} b(\cos \theta) d \omega=b_{0}, \quad 0 \leq b(\cos \theta) \leq b_{1},
$$

where $b_{0}, b_{1}$ are positive constants.

Recently, Ukai-Yang [26] proved that under the assumption A1, the Cauchy problem (1.1)-(1.2) for the Boltzmann equation is well-posed globally in time near a global Maxwellian in function spaces without any regularity condition on the derivatives. To be precise, without loss of generality, let the global Maxwellian $\mathbf{M}(\cdot)$ be

$$
\mathbf{M}(\xi)=\frac{1}{(2 \pi)^{3 / 2}} \exp \left(-\frac{|\xi|^{2}}{2}\right) .
$$

The function space $X_{\beta}$ is defined by

$$
X_{\beta}=L^{2}\left(\mathbb{R}_{x}^{3} \times \mathbb{R}_{\xi}^{3}\right) \cap L^{\infty}\left(\mathbb{R}_{x}^{3} ; L_{\beta}^{\infty}\left(\mathbb{R}_{\xi}^{3}\right)\right),
$$

with norm

$$
\|g\|_{X_{\beta}}=\|g\|_{L^{2}\left(\mathbb{R}_{x}^{3} \times \mathbb{R}_{\xi}^{3}\right)}+\left\|\langle\xi\rangle^{\beta} g\right\|_{L^{\infty}\left(\mathbb{R}_{x}^{3} \times \mathbb{R}_{\xi}^{3}\right)},
$$

where $\langle\xi\rangle=\left(1+|\xi|^{2}\right)^{1 / 2}$ and $g=g(x, \xi)$. Then the following existence result was proved in $[26]$.

Proposition 1.1. Let $\beta>3 / 2$ and the condition $A 1$ hold. There are positive constants $\delta_{0}$ and $C_{0}$ such that if the initial data satisfies

$$
\begin{gathered}
f_{0}(x, \xi)=\mathbf{M}(\xi)+\sqrt{\mathbf{M}(\xi)} u_{0}(x, \xi) \geq 0, \quad x \in \mathbb{R}^{3}, \xi \in \mathbb{R}^{3}, \\
u_{0} \in X_{\beta}, \quad\left\|u_{0}\right\|_{X_{\beta}} \leq \delta_{0},
\end{gathered}
$$

then the Cauchy problem (1.1)-(1.2) has a unique solution

$$
\begin{gathered}
f(t, x, \xi)=\mathbf{M}(\xi)+\sqrt{\mathbf{M}(\xi)} u(t, x, \xi) \geq 0, \quad t \geq 0, x \in \mathbb{R}^{3}, \xi \in \mathbb{R}^{3}, \\
u \in L^{\infty}\left(\mathbb{R}_{+} ; X_{\beta}\right), \quad \sup _{t \geq 0}\|u(t)\|_{X_{\beta}} \leq C_{0}\left\|u_{0}\right\|_{X_{\beta}} .
\end{gathered}
$$


The purpose of this paper is to study the regularity of the solution $f$, or equivalently, the perturbation $u$ obtained in the above proposition. As in the case of small perturbation near vacuum studied by Boudin-Desvillettes [8], we prove that the solution $f$ in Proposition 1.1 can also be written into a sum of two parts in which one corresponds to the free transportation of the initial data with a coefficient decaying exponentially in time and having fractional derivatives in some Sobolev space, while the other one is just regular with locally bounded fractional derivatives. This shows that for the solutions near a global equilibrium, the singularities of the initial data also propagate along the free transportation. To state this theorem, we need one more assumption given below.

A2. The angular part $b(\cdot)$ in the cross section $B(\cdot, \cdot)$ satisfies

$$
\sup _{|y| \leq 1}\left|\frac{\partial b}{\partial y}(y)\right| \leq b_{2}
$$

where $b_{2}>0$ is a constant.

The main result in this paper can be stated as follows.

Theorem 1.1. Let the conditions A1 and A2 hold. Suppose that there is a solution $f$ to the Cauchy problem (1.1)-(1.2) such that

$$
\begin{gathered}
f(t, x, \xi)=\mathbf{M}(\xi)+\sqrt{\mathbf{M}(\xi)} u(t, x, \xi) \geq 0, \quad t \geq 0, x \in \mathbb{R}^{3}, \xi \in \mathbb{R}^{3}, \\
u \in L^{\infty}\left(\mathbb{R}_{+} ; L^{2}\left(\mathbb{R}_{x}^{3} \times \mathbb{R}_{\xi}^{3}\right) \cap L^{\infty}\left(\mathbb{R}_{x}^{3} \times \mathbb{R}_{\xi}^{3}\right)\right) .
\end{gathered}
$$

Then $f$ can be written as

$$
f(t, x, \xi)=f_{0}(x-\xi t, \xi) \Gamma_{1}(t, x, \xi)+\Gamma_{2}(t, x, \xi),
$$

for all $t \geq 0, x \in \mathbb{R}^{3}, \xi \in \mathbb{R}^{3}$, where there exits $\alpha_{0}=\alpha_{0}(\gamma)>0$ defined by

$$
\alpha_{0}(\gamma)= \begin{cases}\frac{1}{25}, & \text { if } \gamma=0, \\ \frac{\gamma}{5(3+2 \gamma)}, & \text { if } 0<\gamma \leq 1,\end{cases}
$$

such that

$$
\Gamma_{1}, \Gamma_{2} \in H_{\text {loc }}^{\alpha}\left(\mathbb{R}_{+} \times \mathbb{R}_{x}^{3} \times \mathbb{R}_{\xi}^{3}\right)
$$

for all $\alpha \in\left(0, \alpha_{0}\right)$. Equivalently, for the perturbation $u$, we have

$$
\begin{gathered}
u(t, x, \xi)=u_{0}(x-\xi t, \xi) \widetilde{\Gamma}_{1}(t, x, \xi)+\widetilde{\Gamma}_{2}(t, x, \xi), \\
\widetilde{\Gamma}_{1}, \widetilde{\Gamma}_{2} \in H_{l o c}^{\alpha}\left(\mathbb{R}_{+} \times \mathbb{R}_{x}^{3} \times \mathbb{R}_{\xi}^{3}\right), \quad 0<\alpha<\alpha_{0} .
\end{gathered}
$$

Remark 1.1. By Proposition 1.1, there exist solutions satisfying (1.3)-(1.4) required in Theorem 1.1. However, for the study of propagation of singularities, so far we don't need the decay of the scaled perturbation $u$ in the velocity variable even though it is needed in the existence theorem, that is, the index $\beta$ in the space $X_{\beta}$ is greater than $\frac{3}{2}$ in the existence theorem while $\beta$ is zero in Theorem 1.1. It would be interesting to find out whether the Cauchy problem (1.1)-(1.2) is well-posed in $X_{\beta}$ when $\beta \leq \frac{3}{2}$.

Remark 1.2. Notice that the index $\alpha_{0}(\gamma)$ obtained in Theorem 1.1 has a jump when $\gamma=0$. This index in the Sobolev space shows the effect of the kinetic part $|z|^{\gamma}$ in the cross section on the regularity in the solutions. The index given here is by no means to be optimal even though it gives some interesting relation between two indices. 
Now let us review some related research to the problem considered here. In the last two decades, there is an enormous literature on the study of regularity properties of solutions to the Boltzmann equation with or without the Grad's angular cutoff assumption. In the non-cutoff case, the solution is more regular than the initial data because the non-cutoff collision operator behaves like a fraction of Laplacian which has regularizing effect on the solution, cf. [2] and references therein. In fact, there are a lot of works and there is a satisfactory theory for the spatially homogeneous Boltzmann equation, cf. [11] and references therein. For example, the $C^{\infty}$ regularization property of weak solutions for the Maxwellian molecule and the regularized hard potentials was proved in [3, 4] by using the Littlewood-Paley decomposition, and the Gevrey regularity for the Maxwellian molecule was obtained in [10, 22]. However, the progress on the spatially inhomogeneous Boltzmann equation without cutoff is much less, cf. [5] and references therein.

On the other hand, in the angular cutoff case, regularity properties of solutions are completely different from those for the non-cutoff potentials. In the cutoff case, the regularities as well as singularities of the initial data propagate in time according to the hyperbolicity of the equation. In the content of the spatially inhomogeneous Boltzmann equation, this kind of propagation for solutions near a vacuum was first studied in [8]. It was shown that the solution at time $t>0$ has the same regularity as the initial data. An extension of this result to the Vlasov-Poisson-Boltzmann system was recently given in [6]. We would also like to mention that the case of the space homogeneous equation has been extensively studied in [23].

Based on the existence result stated in Proposition 1.1, the study in this paper is motivated by the work of [8] for the perturbation of vacuum. What plays the key role in the whole analysis is still the combination of the velocity regularization properties of the positive part in the Boltzmann operator [7, 20, 21, 28] and the spatial regularization properties from the averaging lemma $[9,14]$. However, some differences in the proof for Theorem 1.1 from the one for the result near vacuum can be explained as follows. Under the current consideration, the perturbation does not decay exponentially in the space variable which is the case for the perturbation of vacuum studied in [8]. In fact, the solution studied in [8] satisfies

$$
0 \leq f(t, x, \xi) \leq C_{T} \exp \left(-\frac{1}{2}|x-\xi t|^{2}+|\xi|^{2}\right)
$$

for any $0 \leq t \leq T, x \in \mathbb{R}^{3}, \xi \in \mathbb{R}^{3}$ even though it can be generalized to algebraic decay in the space variable. Now the perturbation of the global Maxwellian still decays exponentially in $\xi$, but it is only bounded in $L^{\infty}\left(\mathbb{R}^{3}\right) \cap L^{2}\left(\mathbb{R}^{3}\right)$ in $x$ variable. Hence, instead of using the direct pointwise bound like (1.10) for the vacuum perturbation, we need to use the uniform bound on the perturbation in the function space (1.4). In addition, only the bounded cross section satisfying

$$
B(z, \cos \theta) \in L^{\infty}\left([-1,1] ; W^{1, \infty}\left(\mathbb{R}^{3}\right)\right),
$$

was considered in $[6,8]$. In this paper, both the hard potentials with angular cutoff and the hard sphere model are considered so that the analysis involves more subtle estimates. Notice that the analysis in this paper can be applied to the study on the perturbation of vacuum provided that the pointwise bound (1.10) is replaced by a weaker assumption, that is,

$$
0 \leq \exp \left(\frac{|\xi|^{2}}{4}\right) f(t, x, \xi) \in L^{\infty}\left([0, T] ; L^{2}\left(\mathbb{R}_{x}^{3} \times \mathbb{R}_{\xi}^{3}\right) \cap L^{\infty}\left(\mathbb{R}_{x}^{3} \times \mathbb{R}_{\xi}^{3}\right)\right)
$$


where the exponential weight can be changed to some algebraic weight $\langle\xi\rangle^{k}$ for some $k$ large enough.

Finally, we would like to mention some other related works on the well-posedness theory of the Cauchy problem for the Boltzmann equation, that is, the global renormalized solution in [12], global solutions in $\mathbb{R}^{3}$ near Maxwellians in [13, 18, 24, 25, 26], global solutions near a vacuum in $[16,17,19]$. Interested reader can find the review paper [27] for more detailed references.

The rest of this paper is arranged as follows. In Section 2, we give the line of proof for Theorem 1.1 and list some basic lemmas. The regularities of the loss term and the gain term are obtained in Section 3 and Section 4, respectively. The proof of Theorem 1.1 is given at the end of Section 4.

Notations. Let $N \geq 1, \ell \geq 0$ be integers and $\Omega \subset \mathbb{R}^{N}$ be an open set with smooth boundary. $L_{k}^{p}(\Omega)$, with $1 \leq p \leq \infty, k \in \mathbb{R}$ denotes the weighted Lebesgue spaces with norms

$$
\|f\|_{L_{k}^{p}(\Omega)}=\left(\int_{\Omega}\langle\xi\rangle^{k p}|f(\xi)|^{p}\right)^{1 / p}, \quad 1 \leq p<\infty
$$

and

$$
\|f\|_{L_{k}^{\infty}(\Omega)}=\sup _{\xi \in \Omega}\langle\xi\rangle^{k}|f(\xi)| .
$$

In addition, $W^{\ell, p}(\Omega), \dot{W}^{\ell, p}(\Omega), 1 \leq p \leq \infty$ denote the usual Sobolev spaces and homogeneous Sobolev spaces respectively, with the convection $H^{\ell}=W^{\ell, 2}, \dot{H}^{\ell}=\dot{W}^{\ell, 2}$. Further, we will use $W^{s, p}(\Omega)$, with $0<s<1,1 \leq p<\infty$ to denote the fractional Sobolev spaces with the norm

$$
\|f\|_{W^{s, p}(\Omega)}=\left(\|f\|_{L^{p}(\Omega)}^{p}+\iint_{\Omega \times \Omega} \frac{|f(\xi)-f(\eta)|^{p}}{|\xi-\eta|^{N+p s}} d \xi d \eta\right)^{1 / p}
$$

Throughout this paper, $C$ denotes a generic constant which may vary from line to line. If the dependence of the constant on some parameter, for example $a$, needs to be specified, then the notation $C_{a}$ will be used. Finally, $B_{R}$ denotes a ball with center at origin and radius $R$.

\section{Mild Form and Basic Lemmas}

Write the solution $f$ to the Cauchy problem (1.1)-(1.2) in the mild form

$$
\begin{aligned}
f(t, x, \xi)= & f_{0}(x-\xi t, \xi) \exp \left(-\int_{0}^{t} L f(\theta, x-\xi \theta, \xi) d \theta\right) \\
& +\int_{0}^{t} Q^{+}(f, f)(s, x-\xi s, \xi) \exp \left(-\int_{s}^{t} L f(\theta, x-\xi \theta, \xi) d \theta\right) d s
\end{aligned}
$$

and set

$$
\begin{aligned}
& \Gamma_{1}=\exp \left(-\int_{0}^{t} L f(\theta, x-\xi \theta, \xi) d \theta\right) \\
& \Gamma_{2}=\int_{0}^{t} Q^{+}(f, f)(s, x-\xi s, \xi) \exp \left(-\int_{s}^{t} L f(\theta, x-\xi \theta, \xi) d \theta\right) d s .
\end{aligned}
$$


As in [8], in order to prove Theorem 1.1 for the regularity of $\Gamma_{1}, \Gamma_{2}$ with fractional Sobolev derivatives, it is equivalent to show

$$
L f, Q^{+}(f, f) \in L_{l o c}^{2}\left(\mathbb{R}_{+} ; H_{l o c}^{\alpha}\left(\mathbb{R}_{x}^{3} \times \mathbb{R}_{\xi}^{3}\right)\right), \quad \forall \alpha \in\left(0, \alpha_{0}\right),
$$

for some $\alpha_{0}>0$. In fact, one has

$$
\begin{aligned}
L f= & A *_{\xi} \mathbf{M}+A *_{\xi}(\sqrt{\mathbf{M}} u), \\
Q^{+}(f, f)= & \mathbf{M} A *_{\xi} \mathbf{M}+Q^{+}(\mathbf{M}, \sqrt{\mathbf{M}} u)+Q^{+}(\sqrt{\mathbf{M}} u, \mathbf{M}) \\
& +Q^{+}(\sqrt{\mathbf{M}} u, \sqrt{\mathbf{M}} u) .
\end{aligned}
$$

Notice that $A *_{\xi} \mathbf{M}, \mathbf{M} A *_{\xi} \mathbf{M}$ depending only on $\xi$ are $C^{1}$-smooth functions. Thus, it suffices to consider the regularity of other terms. Firstly, for the convolution term in $L f$, the velocity regularity follows naturally from that of $A(\cdot)$, whereas the spatial regularity will be obtained by the averaging lemma proved in [9] stated as follows.

Lemma 2.1 ([9]). Let $T>0$ and $f \in C\left([0, T] ; L^{2}\left(\mathbb{R}_{x}^{3} \times \mathbb{R}_{\xi}^{3}\right)\right)$. Suppose

$$
g(t, x, \xi):=\partial_{t} f(t, x, \xi)+\xi \cdot \nabla_{x} f(t, x, \xi) \in L^{2}\left([0, T] \times \mathbb{R}_{x}^{3} \times \mathbb{R}_{\xi}^{3}\right) .
$$

Then for any $\psi \in C_{c}^{\infty}\left(\mathbb{R}_{\xi}^{3}\right)$, the average quantity

$$
\rho_{\psi}(t, x):=\int_{\mathbb{R}^{3}} f(t, x, \xi) \psi(\xi) d \xi
$$

satisfies

$$
\rho_{\psi} \in L^{2}\left([0, T] ; H^{1 / 2}\left(\mathbb{R}_{x}^{3}\right)\right)
$$

In fact, for any $s>1$, we have

$$
\begin{gathered}
\left\|\rho_{\psi}\right\|_{L^{2}\left([0, T] ; H^{1 / 2}\left(\mathbb{R}_{x}^{3}\right)\right)} \leq C_{s}\left(\int_{\mathbb{R}^{3} \times \mathbb{R}^{3}}|f(0, x, \xi)|^{2}|\psi(\xi)|^{2}\langle\xi\rangle^{2 s} d x d \xi\right. \\
\left.+\iiint_{[0, T] \times \mathbb{R}^{3} \times \mathbb{R}^{3}}|g(t, x, \xi)|^{2}|\psi(\xi)|^{2}\langle\xi\rangle^{2 s} d x d \xi d t\right)
\end{gathered}
$$

where $C_{s}$ is a constant depending only on $s$.

Actually, we can apply the above lemma to the following equation for $\sqrt{\mathbf{M}} u$ :

$$
\begin{gathered}
\partial_{t}(\sqrt{\mathbf{M}} u)+\xi \cdot \nabla_{x}(\sqrt{\mathbf{M}} u) \\
=Q^{+}(\mathbf{M}, \sqrt{\mathbf{M}} u)+Q^{+}(\sqrt{\mathbf{M}} u, \mathbf{M})+Q^{+}(\sqrt{\mathbf{M}} u, \sqrt{\mathbf{M}} u) \\
-\mathbf{M} A *_{\xi}(\sqrt{\mathbf{M}} u)-\sqrt{\mathbf{M}} u A *_{\xi} \mathbf{M}-\sqrt{\mathbf{M}} u A *_{\xi}(\sqrt{\mathbf{M}} u) .
\end{gathered}
$$

On the other hand, the terms in $Q^{+}(f, f)$ are regular in the velocity variable by the following lemma proved in [7], which in turn will lead to the regularity in the spatial variable with the help of the velocity mollifier and the aforementioned velocity averaging lemma. 
Lemma 2.2 ([7]). Under conditions $A 1$ and A2, for any $\epsilon>0$, there is a constant $C_{\epsilon}$ depending only on $\epsilon$ such that for any $f, g \in L_{1}^{1}\left(\mathbb{R}_{\xi}^{3}\right) \cap L_{(3+\epsilon) / 2}^{2}\left(\mathbb{R}_{\xi}^{3}\right)$, one has

$$
Q^{+}(f, g) \in \dot{H}^{1}\left(\mathbb{R}_{\xi}^{3}\right),
$$

and

$$
\left\|Q^{+}(f, g)\right\|_{\dot{H}^{1}\left(\mathbb{R}_{\xi}^{3}\right)} \leq C_{\epsilon}\left(b_{1}+b_{2}\right)\|f\|_{L_{(3+\epsilon) / 2}^{2}\left(\mathbb{R}_{\xi}^{3}\right)}\|g\|_{L_{(3+\epsilon) / 2}^{2}\left(\mathbb{R}_{\xi}^{3}\right)} .
$$

Finally, for later use, we list some basic estimates whose proofs can be found in [1, 25].

Lemma 2.3. Let $s>-3, \lambda>0$. There is a constant $C_{s, \lambda}$ depending only on $s, \lambda$, such that for all $\xi \in \mathbb{R}^{3}$, it holds

$$
\int_{\mathbb{R}^{3}}\left|\xi-\xi_{*}\right|^{s} \exp \left(-\lambda\left|\xi_{*}\right|^{2}\right) d \xi_{*} \leq C_{s, \lambda}\langle\xi\rangle^{s} .
$$

Lemma 2.4. Let $f=f(x) \in W_{\text {loc }}^{1, \infty}\left(\mathbb{R}^{3}\right)$. Then $f \in H_{\text {loc }}^{1 / 2}\left(\mathbb{R}^{3}\right)$, and there is a constant $C$ such that for any $R>0$, it holds that

$$
\|f\|_{H^{1 / 2}\left(B_{R}\right)} \leq C R^{\frac{3}{2}}\|f\|_{W^{1, \infty}\left(B_{R}\right)} .
$$

Lemma 2.5. Let $h \in \mathbb{R}^{3}$. Denote the shift operator $\tau_{h}$ by $\tau_{h} f(x)=f(x-h)$. Then for any $f \in H^{1 / 2}\left(\mathbb{R}^{3}\right)$, it holds

$$
\left\|\tau_{h} f-f\right\|_{L^{2}\left(\mathbb{R}^{3}\right)} \leq\|f\|_{\dot{H}^{1 / 2}\left(\mathbb{R}^{3}\right)}|h|^{\frac{1}{2}} .
$$

Lemma 2.6. Let $\psi(\xi)$ be the standard mollifier, and $\psi_{\delta}(\cdot)=\frac{1}{\delta^{3}} \psi(\dot{\bar{\delta}})$, for $\delta>0$. Then for any $f=f(\xi) \in H^{1}\left(\mathbb{R}^{3}\right)$, it holds

$$
\left\|f-\psi_{\delta} *_{\xi} f\right\|_{L^{2}\left(\mathbb{R}^{3}\right)} \leq C_{\psi}\|f\|_{\dot{H}^{1}\left(\mathbb{R}^{3}\right)} \delta,
$$

where $C_{\psi}$ is a constant depending only on $\psi$.

\section{Regularity of $L f$}

Recall the representation (2.1) of $L f$. Firstly, we consider its regularity in the velocity variable.

Lemma 3.1. Under the assumptions of Theorem 1.1, it holds

$$
A *_{\xi}(\sqrt{\mathbf{M}} u) \in L^{2}\left([0, T] \times \mathbb{R}_{x}^{3} ; H^{1 / 2}\left(B_{R_{\xi}}\right)\right),
$$

with

$$
\left\|A *_{\xi}(\sqrt{\mathbf{M}} u)\right\|_{L^{2}\left([0, T] \times \mathbb{R}_{x}^{3} ; H^{1 / 2}\left(B_{R_{\xi}}\right)\right)} \leq C R^{\frac{3}{2}}\langle R\rangle^{\gamma} \sqrt{T}\|u\|_{L^{\infty}\left([0, T] ; L^{2}\left(\mathbb{R}_{x}^{3} \times \mathbb{R}_{\xi}^{3}\right)\right)} .
$$

Proof. By the Hölder inequality and Lemma 2.3, we have

$$
\begin{aligned}
\left|A *_{\xi}(\sqrt{\mathbf{M}} u)\right|^{2} & \leq \int_{\mathbb{R}^{3}} A\left(\xi-\xi_{*}\right)^{2} \mathbf{M}\left(\xi_{*}\right) d \xi_{*} \int_{\mathbb{R}^{3}}\left|u\left(t, x, \xi_{*}\right)\right|^{2} d \xi_{*} \\
& \leq C b_{0}^{2}\langle\xi\rangle^{2 \gamma} \int_{\mathbb{R}^{3}}\left|u\left(t, x, \xi_{*}\right)\right|^{2} d \xi_{*} .
\end{aligned}
$$


Similarly, it holds that

$$
\begin{aligned}
& \left|\nabla_{\xi}\left(A *_{\xi}(\sqrt{\mathbf{M}} u)\right)\right|^{2}=\left|\nabla_{\xi} A *_{\xi}(\sqrt{\mathbf{M}} u)\right|^{2} \\
\leq & C b_{0}^{2}\langle\xi\rangle^{2 \gamma-2} \int_{\mathbb{R}^{3}}\left|u\left(t, x, \xi_{*}\right)\right|^{2} d \xi_{*} \leq C b_{0}^{2} \int_{\mathbb{R}^{3}}\left|u\left(t, x, \xi_{*}\right)\right|^{2} d \xi_{*} .
\end{aligned}
$$

Thus for any $t \geq 0, x \in \mathbb{R}^{3}$, we have

$$
\left\|A *_{\xi}(\sqrt{\mathbf{M}} u)\right\|_{W^{1, \infty}\left(B_{R_{\xi}}\right)} \leq C b_{0}\langle R\rangle^{\gamma}\|u(t, x, \cdot)\|_{L^{2}\left(\mathbb{R}_{\xi}^{3}\right)} .
$$

This together with the imbedding $W_{\text {loc }}^{1, \infty}\left(\mathbb{R}_{\xi}^{3}\right) \hookrightarrow H_{\text {loc }}^{1 / 2}\left(\mathbb{R}_{\xi}^{3}\right)$ stated in Lemma 2.4 completes the proof of the lemma.

As in [8], the regularity in the space variable follows from the velocity averaging lemma.

Lemma 3.2. Under the assumptions of Theorem 1.1, it holds

$$
A *_{\xi}(\sqrt{\mathbf{M}} u) \in L^{2}\left([0, T] \times B_{R_{\xi}} ; H^{1 / 2}\left(\mathbb{R}_{x}^{3}\right)\right),
$$

with

$$
\left\|A *_{\xi}(\sqrt{\mathbf{M}} u)\right\|_{L^{2}\left([0, T] \times B_{R_{\xi}} ; H^{1 / 2}\left(\mathbb{R}_{x}^{3}\right)\right)} \leq C R^{\frac{3}{2}}\langle R\rangle^{\gamma} N_{T}(u),
$$

where

$$
N_{T}(u)=\left\|u_{0}\right\|_{L^{2}\left(\mathbb{R}_{x}^{3} \times \mathbb{R}_{\xi}^{3}\right)}+\sqrt{T}\|u\|_{L^{\infty}\left([0, T] ; L^{2}\left(\mathbb{R}_{x}^{3} \times \mathbb{R}_{\xi}^{3}\right)\right)}\left(1+\|u\|_{L^{\infty}[0, T] \times \mathbb{R}_{x}^{3} \times \mathbb{R}_{\xi}^{3}}\right) .
$$

Proof. Firstly, by applying Lemma 2.1, we can obtain

$$
\begin{aligned}
& \left\|A *_{\xi}(\sqrt{\mathbf{M}} u)\right\|_{L^{2}\left([0, T] \times B_{R_{\xi}} ; H^{1 / 2}\left(\mathbb{R}_{x}^{3}\right)\right)}^{2} \leq \int_{B_{R_{\xi}}}\left\|A *_{\xi}(\sqrt{\mathbf{M}} u)\right\|_{L^{2}\left([0, T] ; H^{1 / 2}\left(\mathbb{R}_{x}^{3}\right)\right)}^{2} d \xi \\
& =\int_{B_{R_{\xi}}}\left\|\rho_{A(\xi-\cdot) e_{\lambda}}\left(\frac{\sqrt{\mathbf{M}} u}{e_{\lambda}}\right)\right\|_{L^{2}\left([0, T] ; H^{1 / 2}\left(\mathbb{R}_{x}^{3}\right)\right)}^{2} d \xi \leq C_{s} \int_{B_{R_{\xi}}}\left[I_{1}(\xi)+I_{2}(\xi)\right] d \xi,
\end{aligned}
$$

where

$$
\begin{aligned}
& I_{1}(\xi)=\iint_{x, \xi_{*}}\left|\frac{\sqrt{\mathbf{M}\left(\xi_{*}\right)} u\left(0, x, \xi_{*}\right)}{e_{\lambda}\left(\xi_{*}\right)}\right|^{2}\left|A\left(\xi-\xi_{*}\right)\right|^{2} e_{\lambda}\left(\xi_{*}\right)^{2}\left\langle\xi_{*}\right\rangle^{2 s} \\
& I_{2}(\xi)=\iiint_{t, x, \xi_{*}}\left|\left(\partial_{t}+\xi_{*} \cdot \nabla_{x}\right) \frac{\sqrt{\mathbf{M}\left(\xi_{*}\right)} u\left(t, x, \xi_{*}\right)}{e_{\lambda}\left(\xi_{*}\right)}\right|^{2}\left|A\left(\xi-\xi_{*}\right)\right|^{2} e_{\lambda}\left(\xi_{*}\right)^{2}\left\langle\xi_{*}\right\rangle^{2 s} .
\end{aligned}
$$

Here and hereafter, $e_{\lambda}$ denotes

$$
e_{\lambda}=e_{\lambda}(\xi)=\exp \left(-\lambda|\xi|^{2}\right)
$$


for $\lambda>0$ to be chosen later. For simplicity, the following multiple integral notions are used

$$
\iint_{x, \xi_{*}}(\cdots)=\iint_{\mathbb{R}^{3} \times \mathbb{R}^{3}}(\cdots) d x d \xi_{*}, \quad \iiint_{t, x, \xi_{*}}(\cdots)=\iint_{[0, T] \times \mathbb{R}^{3} \times \mathbb{R}^{3}}(\cdots) d t d x d \xi_{*} .
$$

We estimate $I_{1}(\xi), I_{2}(\xi)$ as follows. For $I_{1}(\xi)$, notice that

$$
A\left(\xi-\xi_{*}\right) \leq b_{1}\left|\xi-\xi_{*}\right|^{\gamma} \leq b_{1}\left(|\xi|+\left|\xi_{*}\right|\right)^{\gamma} \leq b_{1}\langle\xi\rangle^{\gamma}\left\langle\xi_{*}\right\rangle^{\gamma} .
$$

Define the function $M_{\lambda, \gamma}(\xi)$ by

$$
M_{\lambda, \gamma}(\xi)=\langle\xi\rangle^{\gamma} \exp \left(-\lambda|\xi|^{2}\right),
$$

and the corresponding constant $M_{\lambda, \gamma, \infty}$ by

$$
M_{\lambda, \gamma, \infty}=\sup _{\xi \in \mathbb{R}^{3}} M_{\lambda, \gamma}(\xi) .
$$

Then we have

$$
\begin{aligned}
I_{1}(\xi) & \leq C b_{1}^{2} M_{\lambda, s+\gamma, \infty}^{2}\langle\xi\rangle^{2 \gamma} \iint_{x, \xi_{*}} \exp \left(-\frac{\left|\xi_{*}\right|^{2}}{2}+2 \lambda\left|\xi_{*}\right|^{2}\right)\left|u_{0}\left(x, \xi_{*}\right)\right|^{2} \\
& \leq C b_{1}^{2} M_{\lambda, s+\gamma, \infty}^{2}\langle\xi\rangle^{2 \gamma}\left\|u_{0}\right\|_{L^{2}\left(\mathbb{R}_{x}^{3} \times \mathbb{R}_{\xi}^{3}\right)}^{2}
\end{aligned}
$$

when $\lambda \leq 1 / 4$. Similarly, for $I_{2}(\xi)$, it holds that

$$
I_{2}(\xi) \leq b_{1}^{2} M_{\lambda, s+\gamma, \infty}^{2}\langle\xi\rangle^{2 \gamma} \iint_{t, x, \xi_{*}}\left|\left(\partial_{t}+\xi_{*} \cdot \nabla_{x}\right) \frac{\sqrt{\mathbf{M}\left(\xi_{*}\right)} u\left(t, x, \xi_{*}\right)}{e_{\lambda}\left(\xi_{*}\right)}\right|^{2} .
$$

Recall the equation (2.3) which is satisfied by $\sqrt{\mathbf{M}} u$. We need to estimate the $L^{2}([0, T] \times$ $\left.\mathbb{R}_{x}^{3} \times \mathbb{R}_{\xi}^{3}\right)$ norm for all the terms on the right hand of (2.3). Firstly, for $Q^{+}(\mathbf{M}, \sqrt{\mathbf{M}} u)$, we have

$$
\begin{aligned}
& \frac{\left|Q^{+}(\mathbf{M}, \sqrt{\mathbf{M}} u)\right|}{e_{\lambda}(\xi)} \leq \frac{Q^{+}(\sqrt{\mathbf{M}}, \sqrt{\mathbf{M}}|u|)}{e_{\lambda}(\xi)} \leq \frac{\mathbf{M}(\xi)^{\frac{1}{6}}}{e_{\lambda}(\xi)} Q^{+}\left(\mathbf{M}^{\frac{1}{3}}, \mathbf{M}^{\frac{1}{3}}|u|\right) \\
= & \frac{\mathbf{M}(\xi)^{\frac{1}{6}}}{e_{\lambda}(\xi)} \iint_{\xi_{*}, \omega} B\left(\xi-\xi_{*}, \cos \theta\right) \mathbf{M}\left(\xi^{\prime}\right)^{\frac{1}{3}} \mathbf{M}\left(\xi_{*}^{\prime}\right)^{\frac{1}{3}}\left|u\left(t, x, \xi_{*}^{\prime}\right)\right| \\
\leq & C e_{1 / 12-\lambda}(\xi)\left[\iint_{\xi_{*}, \omega} B\left(\xi-\xi_{*}, \cos \theta\right)^{2} \mathbf{M}(\xi)^{\frac{1}{3}} \mathbf{M}\left(\xi_{*}\right)^{\frac{1}{3}}\right]^{\frac{1}{2}}\left[\iint_{\xi_{*}, \omega} \mathbf{M}\left(\xi^{\prime}\right)^{\frac{1}{3}} \mathbf{M}\left(\xi_{*}^{\prime}\right)^{\frac{1}{3}}\left|u\left(t, x, \xi_{*}^{\prime}\right)\right|^{2}\right]^{\frac{1}{2}} \\
\leq & C b_{1} e_{1 / 12-\lambda}(\xi)\left[\iint_{\xi_{*}, \omega} \mathbf{M}\left(\xi^{\prime}\right)^{\frac{1}{3}} \mathbf{M}\left(\xi_{*}^{\prime}\right)^{\frac{1}{3}} \mid u\left(t, x, \xi_{*}^{\prime}\right)^{2}\right]^{\frac{1}{2}},
\end{aligned}
$$

where we have used

$$
\begin{aligned}
\iint_{\xi_{*}, \omega} B\left(\xi-\xi_{*}, \cos \theta\right)^{2} \mathbf{M}(\xi)^{\frac{1}{3}} \mathbf{M}\left(\xi_{*}\right)^{\frac{1}{3}} & \leq C b_{1}^{2} \int_{\xi_{*}}\langle\xi\rangle^{2 \gamma}\left\langle\xi_{*}\right\rangle^{2 \gamma} \mathbf{M}(\xi)^{\frac{1}{3}} \mathbf{M}\left(\xi_{*}\right)^{\frac{1}{3}} \\
& \leq C b_{1}^{2} M_{1 / 6,2 \gamma, \infty}
\end{aligned}
$$


Similarly, for $Q^{+}(\sqrt{\mathbf{M}} u, \mathbf{M})$ and $Q^{+}(\sqrt{\mathbf{M}} u, \sqrt{\mathbf{M}} u)$, it holds that

$$
\frac{\left|Q^{+}(\sqrt{\mathbf{M}} u, \mathbf{M})\right|}{e_{\lambda}(\xi)} \leq C b_{1} e_{1 / 12-\lambda}(\xi)\left[\iint_{\xi_{*}, \omega} \mathbf{M}\left(\xi^{\prime}\right)^{\frac{1}{3}} \mathbf{M}\left(\xi_{*}^{\prime}\right)^{\frac{1}{3}}\left|u\left(t, x, \xi^{\prime}\right)\right|^{2}\right]^{\frac{1}{2}},
$$

and

$$
\begin{gathered}
\frac{\left|Q^{+}(\sqrt{\mathbf{M}} u, \sqrt{\mathbf{M}} u)\right|}{e_{\lambda}(\xi)} \leq \frac{Q^{+}(\sqrt{\mathbf{M}}|u|, \sqrt{\mathbf{M}})}{e_{\lambda}(\xi)}\|u\|_{L^{\infty}\left([0, T] \times \mathbb{R}_{x}^{3} \times \mathbb{R}_{\xi}^{3}\right)} \\
\leq C b_{1} e_{1 / 12-\lambda}(\xi)\|u\|_{L^{\infty}\left([0, T] \times \mathbb{R}_{x}^{3} \times \mathbb{R}_{\xi}^{3}\right)}\left[\iint_{\xi_{*}, \omega} \mathbf{M}\left(\xi^{\prime}\right)^{\frac{1}{3}} \mathbf{M}\left(\xi_{*}^{\prime}\right)^{\frac{1}{3}}\left|u\left(t, x, \xi^{\prime}\right)\right|^{2}\right]^{\frac{1}{2}} .
\end{gathered}
$$

For the loss terms, straightforward calculation shows that

$$
\begin{aligned}
\frac{\left|\mathbf{M} A *_{\xi}(\sqrt{\mathbf{M}} u)\right|}{e_{\lambda}(\xi)} \leq \frac{\sqrt{\mathbf{M}} A *_{\xi}(\sqrt{\mathbf{M}}|u|)}{e_{\lambda}(\xi)} \leq C b_{1} e_{1 / 12-\lambda}(\xi)\left[\int_{\xi_{*}}\left|u\left(t, x, \xi_{*}\right)\right|^{2}\right]^{\frac{1}{2}}, \\
\frac{\left|\sqrt{\mathbf{M}} u A *_{\xi} \mathbf{M}\right|}{e_{\lambda}(\xi)} \leq C b_{1} e_{1 / 12-\lambda}(\xi)|u(t, x, \xi)|,
\end{aligned}
$$

and

$$
\frac{\left|\sqrt{\mathbf{M}} u A *_{\xi}(\sqrt{\mathbf{M}} u)\right|}{e_{\lambda}(\xi)} \leq C b_{1} e_{1 / 12-\lambda}(\xi)\|u\|_{L^{\infty}\left([0, T] \times \mathbb{R}_{x}^{3} \times \mathbb{R}_{\xi}^{3}\right)}|u(t, x, \xi)| .
$$

Therefore, by combining all the above estimates, we have

$$
\begin{aligned}
& \iiint_{t, x, \xi}\left|\left(\partial_{t}+\xi \cdot \nabla_{x}\right) \frac{\sqrt{\mathbf{M}(\xi)} u(t, x, \xi)}{e_{\lambda}(\xi)}\right|^{2} \\
& \leq C b_{1}^{2}\left(1+\|u\|_{L^{\infty}\left([0, T] \times \mathbb{R}_{x}^{3} \times \mathbb{R}_{\xi}^{3}\right)}^{2}\right) \times \iint_{t, x, \xi, \xi_{*}, \omega} \ldots e_{1 / 6-2 \lambda}(\xi) \mathbf{M}\left(\xi^{\prime}\right)^{\frac{1}{3}} \mathbf{M}\left(\xi_{*}^{\prime}\right)^{\frac{1}{3}}\left(\left|u\left(t, x, \xi_{*}^{\prime}\right)\right|^{2}+\left|u\left(t, x, \xi^{\prime}\right)\right|^{2}\right) \\
& \quad+C b_{1}^{2}\left(1+\|u\|_{L^{\infty}\left([0, T] \times \mathbb{R}_{x}^{3} \times \mathbb{R}_{\xi}^{3}\right)}^{2}\right) \iiint_{t, x, \xi} e_{1 / 6-2 \lambda}(\xi)|u(t, x, \xi)|^{2}+C b_{1}^{2} \iiint \int_{t, x, \xi, \xi_{*}} e_{1 / 6-2 \lambda}(\xi)\left|u\left(t, x, \xi_{*}\right)\right|^{2} .
\end{aligned}
$$

Thus by choosing $\lambda=1 / 24$ and further making the change of variable $\left(\xi, \xi_{*}\right) \rightarrow\left(\xi^{\prime}, \xi_{*}^{\prime}\right)$, the above inequality together with (3.6) yield

$$
I_{2}(\xi) \leq C b_{1}^{2} T M_{\lambda, s+\gamma, \infty}^{2}\langle\xi\rangle^{2 \gamma}\|u\|_{L^{\infty}\left([0, T] ; L^{2}\left(\mathbb{R}_{x}^{3} \times \mathbb{R}_{\xi}^{3}\right)\right)}^{2}\left(1+\|u\|_{L^{\infty}\left([0, T] \times \mathbb{R}_{x}^{3} \times \mathbb{R}_{\xi}^{3}\right)}^{2}\right) .
$$

Putting (3.5) and (3.7) into (3.2) gives the desired estimate. This completes the proof of the lemma.

Corollary 3.1. Under the assumptions of Theorem 1.1, it holds

$$
\begin{gathered}
A *_{\xi}(\sqrt{\mathbf{M}} u) \in L^{2}\left([0, T] ; H^{1 / 2}\left(\mathbb{R}_{x}^{3} \times B_{R_{\xi}}\right)\right), \\
0 \leq L f \in L^{2}\left([0, T] ; H_{l o c}^{1 / 2}\left(\mathbb{R}_{x}^{3} \times \mathbb{R}_{\xi}^{3}\right)\right) \cap L^{\infty}\left([0, T] \times \mathbb{R}_{x}^{3} ; L_{l o c}^{\infty}\left(\mathbb{R}_{\xi}^{3}\right)\right) .
\end{gathered}
$$




\section{Regularity of $Q^{+}(f, f)$}

Recall the expansion (2.2) for $Q^{+}(f, f)$. For simplicity, denote $S_{1}^{Q}=Q^{+}(\mathbf{M}, \sqrt{\mathbf{M}} u), \quad S_{2}^{Q}=Q^{+}(\sqrt{\mathbf{M}} u, \mathbf{M}), \quad S_{3}^{Q}=Q^{+}(\sqrt{\mathbf{M}} u, \sqrt{\mathbf{M}} u)$.

The regularity in the velocity variable for each term above follows essentially from smoothness of the operator $Q^{+}(\cdot, \cdot)$.

Lemma 4.1. Under the conditions of Theorem 1.1, for each $i=1,2,3$, it holds that

$$
S_{i}^{Q} \in L^{2}\left([0, T] \times \mathbb{R}_{x}^{3} ; H^{1}\left(\mathbb{R}_{\xi}^{3}\right)\right) \cap L^{\infty}\left([0, T] \times \mathbb{R}_{x}^{3} ; H^{1}\left(\mathbb{R}_{\xi}^{3}\right)\right),
$$

and

$$
\begin{aligned}
& \left\|S_{i}^{Q}\right\|_{L^{2}\left([0, T] \times \mathbb{R}_{x}^{3} ; H^{1}\left(\mathbb{R}_{\xi}^{3}\right)\right)} \leq C\left(b_{1}+b_{2}\right) \sqrt{T}\|u\|_{L^{\infty}\left([0, T] ; L^{2}\left(\mathbb{R}_{x}^{3} \times \mathbb{R}_{\xi}^{3}\right)\right)}\left(1+\|u\|_{L^{\infty}\left([0, T] \times \mathbb{R}_{x}^{3} \times \mathbb{R}_{\xi}^{3}\right)}\right), \\
& \left\|S_{i}^{Q}\right\|_{L^{\infty}\left([0, T] \times \mathbb{R}_{x}^{3} ; H^{1}\left(\mathbb{R}_{\xi}^{3}\right)\right)} \leq C\left(b_{1}+b_{2}\right) \sqrt{T}\|u\|_{L^{\infty}\left([0, T] \times \mathbb{R}_{x}^{3} \times \mathbb{R}_{\xi}^{3}\right)}\left(1+\|u\|_{L^{\infty}\left([0, T] \times \mathbb{R}_{x}^{3} \times \mathbb{R}_{\xi}^{3}\right)}\right) .
\end{aligned}
$$

Proof. From the proof of Lemma 3.2, it is straightforward to show that

$$
S_{i}^{Q} \in L^{2}\left([0, T] \times \mathbb{R}_{x}^{3} \times \mathbb{R}_{\xi}^{3}\right) \cap L^{\infty}\left([0, T] \times \mathbb{R}_{x}^{3} ; L^{2}\left(\mathbb{R}_{\xi}^{3}\right)\right) .
$$

Then, it follows from Lemma 2.2 that

$$
\begin{aligned}
&\left\|S_{i}^{Q}\right\|_{\dot{H}^{1}\left(\mathbb{R}_{\xi}^{3}\right)} \leq C_{\epsilon}\left(b_{1}+b_{2}\right)\|\mathbf{M}\|_{L_{(3+\epsilon) / 2}^{2}}\|\sqrt{\mathbf{M}} u\|_{L_{(3+\epsilon) / 2}^{2}}, i=1,2, \\
&\left\|S_{3}^{Q}\right\|_{\dot{H}^{1}\left(\mathbb{R}_{\xi}^{3}\right)} \leq C_{\epsilon}\left(b_{1}+b_{2}\right)\|\sqrt{\mathbf{M}} u\|_{L_{(3+\epsilon) / 2}^{2}}^{2}
\end{aligned}
$$

where $\epsilon>0$ is fixed. Thus, taking $L^{2}\left([0, T] \times \mathbb{R}_{x}^{3}\right)$ or $L^{\infty}\left([0, T] \times \mathbb{R}_{x}^{3}\right)$ norms on both sides of the above inequalities gives the desired estimates. This completes the proof of the lemma.

Next, we consider the spatial regularity of $S_{i}^{Q}$. In order to use the velocity averaging lemma, as in [8], we use the mollifier to construct some velocity averaged quantities. For this, let $\phi \in C_{c}^{\infty}\left(\mathbb{R}_{\xi}^{3}\right)$ and consider the integral

$$
\begin{aligned}
& \int_{\mathbb{R}^{3}} S_{1}^{Q}(t, x, \xi) \phi(\xi) d \xi=\iiint_{\xi, \xi_{*}, \omega} \mathbf{M}\left(\xi^{\prime}\right) \sqrt{\mathbf{M}\left(\xi_{*}^{\prime}\right)} u\left(t, x, \xi_{*}^{\prime}\right) \phi(\xi) B\left(\xi-\xi_{*}, \cos \theta\right) \\
& =\iint_{\xi, \xi_{*}} \mathbf{M}(\xi) \sqrt{\mathbf{M}\left(\xi_{*}\right)} u\left(t, x, \xi_{*}\right) Z^{\phi}\left(\xi, \xi_{*}\right),
\end{aligned}
$$

where

$$
Z^{\phi}\left(\xi, \xi_{*}\right)=\int_{\omega} B\left(\xi-\xi_{*}, \cos \theta\right) \phi\left(\xi-\left(\xi-\xi_{*}\right) \cdot \omega \omega\right) d \omega .
$$

Similarly, corresponding to $S_{2}^{Q}, S_{3}^{Q}$, set

$$
\begin{aligned}
\int_{\mathbb{R}^{3}} S_{2}^{Q}(t, x, \xi) \phi(\xi) d \xi & =\iint_{\xi, \xi_{*}} \sqrt{\mathbf{M}(\xi)} u(t, x, \xi) \mathbf{M}\left(\xi_{*}\right) Z^{\phi}\left(\xi, \xi_{*}\right), \\
\int_{\mathbb{R}^{3}} S_{3}^{Q}(t, x, \xi) \phi(\xi) d \xi & =\iint_{\xi, \xi_{*}} \sqrt{\mathbf{M}(\xi)} u(t, x, \xi) \sqrt{\mathbf{M}\left(\xi_{*}\right)} u\left(t, x, \xi_{*}\right) Z^{\phi}\left(\xi, \xi_{*}\right) .
\end{aligned}
$$

The following lemma is about the pointwise estimates on $Z^{\phi}$. 
Lemma 4.2. Under the assumptions $A 1$ and $A 2$, for any $\xi, \xi_{*}$, it holds that

$$
\left|Z^{\phi}\left(\xi, \xi_{*}\right)\right| \leq C b_{1}\|\phi\|_{L \infty\left(\mathbb{R}^{3}\right)}\left|\xi-\xi_{*}\right|^{\gamma} .
$$

Furthermore, for any $\eta, \eta_{*}$, it holds that for $\gamma=0$,

$$
\left|Z^{\phi}\left(\xi, \xi_{*}\right)-Z^{\phi}\left(\eta, \eta_{*}\right)\right| \leq C\left(b_{1}+b_{2}\right)\|\phi\|_{W^{1, \infty}\left(\mathbb{R}^{3}\right)}\left(|\xi-\eta|+\left|\xi_{*}-\eta_{*}\right|\right),
$$

and for $0<\gamma \leq 1$,

$$
\begin{aligned}
\left|Z^{\phi}\left(\xi, \xi_{*}\right)-Z^{\phi}\left(\eta, \eta_{*}\right)\right| \leq & C\left(b_{1}+b_{2}\right)\|\phi\|_{W^{1, \infty}\left(\mathbb{R}^{3}\right)}\left|\xi-\xi_{*}\right|^{\gamma}\left(|\xi-\eta|+\left|\xi_{*}-\eta_{*}\right|\right) \\
& +C b_{1}\|\phi\|_{L^{\infty}\left(\mathbb{R}^{3}\right)}\left(|\xi-\eta|^{\gamma}+\left|\xi_{*}-\eta_{*}\right|^{\gamma}\right) .
\end{aligned}
$$

Proof. By A1 and A2, (4.4) and (4.5) follow from straightforward calculations. For (4.6), $Z^{\phi}$ is rewritten as

$$
Z^{\phi}\left(\xi, \xi_{*}\right)=\left|\xi-\xi_{*}\right|^{\gamma} Y^{\phi}\left(\xi, \xi_{*}\right)
$$

where

$$
Y^{\phi}\left(\xi, \xi_{*}\right):=\int_{\omega} b(\cos \theta) \phi\left(\xi-\left(\xi-\xi_{*}\right) \cdot \omega \omega\right) d \omega
$$

Notice that $Y^{\phi}\left(\xi, \xi_{*}\right)$ enjoys the same estimate as in (4.5) and

$$
|| \xi-\left.\xi_{*}\right|^{\gamma}-\left|\eta-\eta_{*}\right|^{\gamma}|\leq|\left|\xi-\xi_{*}\right|-\left|\eta-\eta_{*}\right|^{\gamma} \leq|\xi-\eta|^{\gamma}+\left|\xi_{*}-\eta_{*}\right|^{\gamma} .
$$

Hence (4.6) holds. This completes the proof of the lemma.

We now show that the velocity averaged functions given by (4.1), (4.2) and (4.3) are regular in the space variable.

Lemma 4.3. Let the conditions A1, A2 hold. For any $|h| \leq 1$ and each $i=1,2,3$, we have

$$
\begin{gathered}
\iint_{[0, T] \times \mathbb{R}^{3}} d t d x\left|\int_{\mathbb{R}^{3}} S_{i}^{Q}(t, x+h, \xi) \phi(\xi) d \xi-\int_{\mathbb{R}^{3}} S_{i}^{Q}(t, x, \xi) \phi(\xi) d \xi\right|^{2} \\
\leq C K_{T}(u)^{2}\|\phi\|_{W^{1, \infty}\left(\mathbb{R}^{3}\right)}^{2} P_{\gamma}(h)
\end{gathered}
$$

where

$$
K_{T}(u)=N_{T}(u)\left(1+\|u\|_{L^{\infty}\left([0, T] \times \mathbb{R}_{x}^{3} \times \mathbb{R}_{\xi}^{3}\right)}\right)
$$

with $N_{T}(u)$ defined by (3.1), and $P_{\gamma}(h)$ is defined by

$$
P_{\gamma}(h)= \begin{cases}|h|^{\frac{2}{5}}, & \text { if } \gamma=0, \\ |h|^{\frac{2 \gamma}{3+2 \gamma}}, & \text { if } 0<\gamma \leq 1\end{cases}
$$


Proof. Firstly, consider the case of $i=1$. Rewrite the averaged function (4.1) by

$$
\begin{aligned}
& \int_{\mathbb{R}^{3}} S_{1}^{Q}(t, x, \xi) \phi(\xi) d \xi=J_{1}+J_{2}, \\
& J_{1}=\iint_{\xi, \xi_{*}} \mathbf{M}(\xi) \sqrt{\mathbf{M}\left(\xi_{*}\right)} u\left(t, x, \xi_{*}\right) \iint_{\eta, \eta_{*}} Z\left(\eta, \eta_{*}\right) \psi_{\epsilon}(\xi-\eta) \psi_{\epsilon}\left(\xi_{*}-\eta_{*}\right), \\
& J_{2}=\iint_{\xi, \xi_{*}} \mathbf{M}(\xi) \sqrt{\mathbf{M}\left(\xi_{*}\right)} u\left(t, x, \xi_{*}\right) \iint_{\eta, \eta_{*}}\left[Z\left(\xi, \xi_{*}\right)-Z\left(\eta, \eta_{*}\right)\right] \psi_{\epsilon}(\xi-\eta) \psi_{\epsilon}\left(\xi_{*}-\eta_{*}\right),
\end{aligned}
$$

where $\psi(\cdot)$ is a standard mollifier, and $\psi_{\epsilon}(\cdot)=\frac{1}{\epsilon^{3}} \psi(\dot{\bar{\epsilon}})$, with $\epsilon>0$ to be determined later. Furthermore, $J_{1}$ can be rewritten as

$$
J_{1}=\iint_{\eta, \eta_{*}} Z\left(\eta, \eta_{*}\right) \rho_{\psi_{\epsilon}\left(\cdot-\eta_{*}\right)}(\sqrt{\mathbf{M}} u)(t, x) \int_{\xi} \mathbf{M}(\xi) \psi_{\epsilon}(\xi-\eta) .
$$

Then one has

$$
\iint_{t, x}\left|\tau_{h} J_{1}-J_{1}\right|^{2}=\iint_{t, x}\left|\iint_{\eta, \eta_{*}} Z\left(\eta, \eta_{*}\right)\left[\left(\tau_{h}-I d\right) \rho_{\psi_{\epsilon}\left(\cdot-\eta_{*}\right)}(\sqrt{\mathbf{M}} u)\right](t, x) \int_{\xi} \mathbf{M}(\xi) \psi_{\epsilon}(\xi-\eta)\right|^{2} .
$$

From Lemma 4.2, we have

$$
\begin{aligned}
\left|Z\left(\eta, \eta_{*}\right)\right| & \leq C\|\psi\|_{L^{\infty}\left(\mathbb{R}^{3}\right)}\left|\eta-\eta_{*}\right|^{\gamma} \\
& \leq C\|\psi\|_{L^{\infty}\left(\mathbb{R}^{3}\right)}\left(|\eta-\xi|^{\gamma}+\left|\xi_{*}-\eta_{*}\right|^{\gamma}+|\xi|^{\gamma}+\left|\xi_{*}\right|^{\gamma}\right) \\
& \leq C\|\psi\|_{L^{\infty}\left(\mathbb{R}^{3}\right)}\left(2 \epsilon^{\gamma}+|\xi|^{\gamma}+\left|\xi_{*}\right|^{\gamma}\right),
\end{aligned}
$$

for any $|\xi-\eta| \leq \epsilon$ and any $\left|\xi_{*}-\eta_{*}\right| \leq \epsilon$. Therefore, we obtain

$$
\iint_{t, x}\left|\tau_{h} J_{1}-J_{1}\right|^{2} \leq C b_{1}^{2}\|\phi\|_{L^{\infty}\left(\mathbb{R}^{3}\right)}^{2}\left(\epsilon^{2 \gamma} J_{11}+J_{12}+J_{13}\right)
$$

where

$$
\begin{aligned}
& J_{11}=\iint_{t, x}\left|\int_{\eta_{*}}\right|\left[\left(\tau_{h}-I d\right) \rho_{\psi_{\epsilon}\left(-\eta_{*}\right)}(\sqrt{\mathbf{M}} u)\right](t, x)\left|\iint_{\xi, \eta} \mathbf{M}(\xi) \psi_{\epsilon}(\xi-\eta)\right|^{2}, \\
& J_{12}=\left.\left.\iint_{t, x}\left|\int_{\eta_{*}}\right|\left[\left(\tau_{h}-I d\right) \rho_{\psi_{\epsilon}\left(\cdot-\eta_{*}\right)}(\sqrt{\mathbf{M}} u)\right](t, x)\left|\iint_{\xi, \eta}\right| \xi\right|^{\gamma} \mathbf{M}(\xi) \psi_{\epsilon}(\xi-\eta)\right|^{2}, \\
& J_{13}=\iint_{t, x}\left|\int_{\eta_{*}}\right|\left[\left(\tau_{h}-I d\right) \rho_{\psi_{\epsilon}\left(\cdot-\eta_{*}\right)}\left(\sqrt{\mathbf{M}}|\xi|^{\gamma} u\right)\right](t, x)\left|\iint_{\xi, \eta} \mathbf{M}(\xi) \psi_{\epsilon}(\xi-\eta)\right|^{2} .
\end{aligned}
$$

In the following, we will only estimate $J_{11}$ because $J_{12}$ and $J_{13}$ can be estimated similarly. In $J_{11}$, the integral over $\eta, \xi$ is bounded uniformly in $\epsilon$, that is,

$$
\iint_{\eta, \xi} \mathbf{M}(\xi) \psi_{\epsilon}(\xi-\eta)=\int_{\xi} \mathbf{M}(\xi) \leq C .
$$


Let $\lambda>0$ be a small constant to be determined later. Then it follows from the Hölder inequality and Lemma 2.5 that

$$
\begin{aligned}
J_{11} & \leq \iint_{t, x}\left|\int_{\eta_{*}}\right|\left[\left(\tau_{h}-I d\right) \rho_{\psi_{\epsilon}\left(\cdot-\eta_{*}\right)}(\sqrt{\mathbf{M}} u)\right](t, x)||^{2} \\
& \leq \iint_{t, x} \int_{\eta_{*}} \exp \left(-\lambda\left|\eta_{*}\right|^{2}\right) \int_{\eta_{*}} \exp \left(\lambda\left|\eta_{*}\right|^{2}\right)\left|\left[\left(\tau_{h}-I d\right) \rho_{\psi_{\epsilon}\left(\cdot-\eta_{*}\right)}(\sqrt{\mathbf{M}} u)\right](t, x)\right|^{2} \\
& \leq \frac{|h|}{\lambda^{3 / 2}} \int_{\eta_{*}} \exp \left(\lambda\left|\eta_{*}\right|^{2}\right)\left\|\rho_{\psi_{\epsilon}\left(\cdot-\eta_{*}\right) e_{\lambda}}\left(\frac{\sqrt{\mathbf{M}} u}{e_{\lambda}}\right)\right\|_{L^{2}\left([0, T] ; \dot{H}^{1 / 2}\left(\mathbb{R}^{3}\right)\right)}^{2} .
\end{aligned}
$$

This together with Lemma 2.1 give

$$
\begin{aligned}
J_{11} \leq & \frac{C_{s}|h|}{\lambda^{3 / 2}} \int_{\eta_{*}} \exp \left(\lambda\left|\eta_{*}\right|^{2}\right)\left(\iint_{x, \xi_{*}}\left|\frac{\sqrt{\mathbf{M}}\left(\xi_{*}\right) u_{0}\left(x, \xi_{*}\right)}{e_{\lambda}\left(\xi_{*}\right)}\right|^{2} \psi_{\epsilon}\left(\xi_{*}-\eta_{*}\right)^{2} e_{\lambda}\left(\xi_{*}\right)^{2}\left\langle\xi_{*}\right\rangle^{2 s}\right. \\
& \left.+\iiint_{t, x, \xi_{*}}\left|\left(\partial_{t}+\xi_{*} \cdot \nabla_{x}\right) \frac{\sqrt{\mathbf{M}}\left(\xi_{*}\right) u\left(t, x, \xi_{*}\right)}{e_{\lambda}\left(\xi_{*}\right)}\right|^{2} \psi_{\epsilon}\left(\xi_{*}-\eta_{*}\right)^{2} e_{\lambda}\left(\xi_{*}\right)^{2}\left\langle\xi_{*}\right\rangle^{2 s}\right) .
\end{aligned}
$$

Notice that

$$
\exp \left(\lambda\left|\eta_{*}\right|^{2}\right) \leq \exp \left(2 \lambda\left|\xi_{*}-\eta_{*}\right|^{2}+2 \lambda\left|\xi_{*}\right|^{2}\right) \leq \exp \left(2 \lambda \epsilon^{2}+2 \lambda\left|\xi_{*}\right|^{2}\right),
$$

holds for any $\left|\xi_{*}-\eta_{*}\right| \leq \epsilon$, and

$$
\int_{\eta_{*}} \psi_{\epsilon}\left(\xi_{*}-\eta_{*}\right)^{2}=\int_{\mathbb{R}^{3}} \psi_{\epsilon}(\xi)^{2} d \xi \leq \frac{C_{\psi}}{\epsilon^{3}},
$$

where $C_{\psi}$ is a constant depending only on $\psi$. Putting these estimates into (4.14) yields

$$
\begin{aligned}
J_{11} \leq & \frac{C_{s} C_{\psi} \exp \left(2 \lambda \epsilon^{2}\right)|h|}{\lambda^{3 / 2} \epsilon^{3}}\left(\iint_{x, \xi_{*}}\left|\frac{\sqrt{\mathbf{M}}\left(\xi_{*}\right) u_{0}\left(x, \xi_{*}\right)}{e_{2 \lambda}\left(\xi_{*}\right)}\right|^{2} e_{\lambda}\left(\xi_{*}\right)^{2}\left\langle\xi_{*}\right\rangle^{2 s}\right. \\
& \left.+\iiint_{t, x, \xi_{*}}\left|\left(\partial_{t}+\xi_{*} \cdot \nabla_{x}\right) \frac{\sqrt{\mathbf{M}}\left(\xi_{*}\right) u\left(t, x, \xi_{*}\right)}{e_{2 \lambda}\left(\xi_{*}\right)}\right|^{2} e_{\lambda}\left(\xi_{*}\right)^{2}\left\langle\xi_{*}\right\rangle^{2 s}\right) .
\end{aligned}
$$

As for the estimates on $I_{1}(\xi), I_{2}(\xi)$ given in (3.3) and (3.4), we can suitably choose some small constant $\lambda>0$ such that

$$
\begin{aligned}
J_{11} \leq & \frac{C_{s} C_{\psi} \exp \left(2 \lambda \epsilon^{2}\right)|h|}{\lambda^{3 / 2} \epsilon^{3}}\left[M_{\lambda, s, \infty}^{2}\left\|u_{0}\right\|_{L^{2}\left(\mathbb{R}_{x}^{3} \times \mathbb{R}_{\xi}^{3}\right)}\right. \\
& \left.+b_{1}^{2} M_{\lambda, s, \infty}^{2} T\|u\|_{L^{\infty}\left([0, T] ; L^{2}\left(\mathbb{R}_{x}^{3} \times \mathbb{R}_{\xi}^{3}\right)\right)}\left(1+\|u\|_{L^{\infty}[0, T] \times \mathbb{R}_{x}^{3} \times \mathbb{R}_{\xi}^{3}}\right)\right] \\
\leq & C_{\psi} N_{T}(u)^{2} \frac{\exp \left(2 \lambda \epsilon^{2}\right)|h|}{\epsilon^{3}}
\end{aligned}
$$


where $N_{T}(u)$ is defined by (3.1). Similarly, it holds that

$$
J_{12}, J_{13} \leq C_{\psi} N_{T}(u)^{2} \frac{\exp \left(2 \lambda \epsilon^{2}\right)|h|}{\epsilon^{3}} .
$$

Therefore, (4.13) gives

$$
\iint_{t, x}\left|\tau_{h} J_{1}-J_{1}\right|^{2} \leq C_{\psi}\|\phi\|_{L^{\infty}\left(\mathbb{R}^{3}\right)}^{2} N_{T}(u)^{2} \frac{\exp \left(2 \lambda \epsilon^{2}\right)\left(\epsilon^{2 \gamma}+1\right)|h|}{\epsilon^{3}} .
$$

Now we turn to the estimates on the following integral for $J_{2}$ :

$$
\begin{aligned}
\iint_{t, x}\left|\tau_{h} J_{2}-J_{2}\right|^{2}=\iint_{t, x} & \mid \iint_{\xi, \xi_{*}} \mathbf{M}(\xi) \sqrt{\mathbf{M}\left(\xi_{*}\right)}\left[u\left(t, x+h, \xi_{*}\right)-u\left(t, x, \xi_{*}\right)\right] \\
& \times\left.\iint_{\eta, \eta_{*}}\left[Z\left(\xi, \xi_{*}\right)-Z\left(\eta-\eta_{*}\right)\right] \psi_{\epsilon}(\xi-\eta) \psi_{\epsilon}\left(\xi_{*}-\eta_{*}\right)\right|^{2} .
\end{aligned}
$$

When $\gamma=0$, by (4.5), we have

$$
\begin{aligned}
& \iint_{\eta, \eta_{*}}\left|Z\left(\xi, \xi_{*}\right)-Z\left(\eta-\eta_{*}\right)\right| \psi_{\epsilon}(\xi-\eta) \psi_{\epsilon}\left(\xi_{*}-\eta_{*}\right) \\
& \leq C b_{1}\|\phi\|_{W^{1, \infty}\left(\mathbb{R}^{3}\right)}\left(\iint_{\eta, \eta_{*}}|\xi-\eta| \psi_{\epsilon}(\xi-\eta) \psi_{\epsilon}\left(\xi_{*}-\eta_{*}\right)+\iint_{\eta, \eta_{*}}\left|\xi_{*}-\eta_{*}\right| \psi_{\epsilon}(\xi-\eta) \psi_{\epsilon}\left(\xi_{*}-\eta_{*}\right)\right) \\
& \leq C b_{1}\|\phi\|_{W^{1, \infty}\left(\mathbb{R}^{3}\right)}\left(\int_{\eta}|\xi-\eta| \psi_{\epsilon}(\xi-\eta) \int_{\eta_{*}} \psi_{\epsilon}\left(\xi_{*}-\eta_{*}\right)+\int_{\eta} \psi_{\epsilon}(\xi-\eta) \int_{\eta_{*}}\left|\xi_{*}-\eta_{*}\right| \psi_{\epsilon}\left(\xi_{*}-\eta_{*}\right)\right) \\
& \leq C_{\psi} b_{1}\|\phi\|_{W^{1, \infty}\left(\mathbb{R}^{3}\right)} \epsilon .
\end{aligned}
$$

When $0<\gamma \leq 1$, similarly, it follows from (4.6) that

$$
\begin{aligned}
& \iint_{\eta, \eta_{*}}\left|Z\left(\xi, \xi_{*}\right)-Z\left(\eta-\eta_{*}\right)\right| \psi_{\epsilon}(\xi-\eta) \psi_{\epsilon}\left(\xi_{*}-\eta_{*}\right) \\
& \leq C b_{1}\|\phi\|_{W^{1, \infty}\left(\mathbb{R}^{3}\right)}\left|\xi-\xi_{*}\right|^{\gamma} \iint_{\eta, \eta_{*}}\left(|\xi-\eta|+\left|\xi_{*}-\eta_{*}\right|\right) \psi_{\epsilon}(\xi-\eta) \psi_{\epsilon}\left(\xi_{*}-\eta_{*}\right) \\
& \quad+C b_{1}\|\phi\|_{L^{\infty}\left(\mathbb{R}^{3}\right)} \iint_{\eta, \eta_{*}}\left(|\xi-\eta|^{\gamma}+\left|\xi_{*}-\eta_{*}\right|^{\gamma}\right) \psi_{\epsilon}(\xi-\eta) \psi_{\epsilon}\left(\xi_{*}-\eta_{*}\right) \\
& \leq C_{\psi} b_{1}\|\phi\|_{W^{1, \infty}\left(\mathbb{R}^{3}\right)}\left(\left|\xi-\xi_{*}\right|^{\gamma} \epsilon+\epsilon^{\gamma}\right) .
\end{aligned}
$$

Thus, for $0 \leq \gamma \leq 1$, it holds that

$$
\iint_{\eta, \eta_{*}}\left|Z\left(\xi, \xi_{*}\right)-Z\left(\eta-\eta_{*}\right)\right| \psi_{\epsilon}(\xi-\eta) \psi_{\epsilon}\left(\xi_{*}-\eta_{*}\right) \leq C_{\psi} b_{1}\|\phi\|_{W^{1, \infty}\left(\mathbb{R}^{3}\right)}\left(\left|\xi-\xi_{*}\right|^{\gamma} \epsilon+\delta_{\gamma} \epsilon^{\gamma}\right),
$$


where $\delta_{\gamma}=0$ if $\gamma=0$ and $\delta_{\gamma}=1$ if $0<\gamma \leq 1$. Putting the above estimate into (4.16) gives

$$
\begin{aligned}
& \iint_{t, x}\left|\tau_{h} J_{2}-J_{2}\right|^{2} \leq C_{\psi} b_{1}^{2}\|\phi\|_{W^{1, \infty}\left(\mathbb{R}^{3}\right)}^{2} \epsilon^{2} \\
& \quad \times \iint_{t, x}\left|\iint_{\xi, \xi_{*}}\right| \xi-\left.\left.\xi_{*}\right|^{\gamma} \mathbf{M}(\xi) \sqrt{\mathbf{M}\left(\xi_{*}\right)}\left(\left|u\left(t, x+h, \xi_{*}\right)\right|+\left|u\left(t, x, \xi_{*}\right)\right|\right)\right|^{2} \\
& \quad+C_{\psi} b_{1}^{2}\|\phi\|_{W^{1, \infty}\left(\mathbb{R}^{3}\right)}^{2} \delta_{\gamma} \epsilon^{2 \gamma} \iint_{t, x}\left|\iint_{\xi, \xi_{*}} \mathbf{M}(\xi) \sqrt{\mathbf{M}\left(\xi_{*}\right)}\left(\left|u\left(t, x+h, \xi_{*}\right)\right|+\left|u\left(t, x, \xi_{*}\right)\right|\right)\right|^{2} .
\end{aligned}
$$

Then Hölder inequality yields

$$
\iint_{t, x}\left|\tau_{h} J_{2}-J_{2}\right|^{2} \leq C_{\psi}\|\phi\|_{W^{1, \infty}\left(\mathbb{R}^{3}\right)}^{2} N_{T}(u)^{2}\left(\epsilon^{2}+\delta_{\gamma} \epsilon^{2 \gamma}\right) .
$$

Now we can complete the proof for (4.7) when $i=1$. In fact, combining (4.15) and (4.17) gives

$$
\begin{aligned}
& \iint_{[0, T] \times \mathbb{R}^{3}} d t d x\left|\int_{\mathbb{R}^{3}} S_{1}^{Q}(t, x+h, \xi) \phi(\xi) d \xi-\int_{\mathbb{R}^{3}} S_{1}^{Q}(t, x, \xi) \phi(\xi) d \xi\right|^{2} \\
& \leq 2 \iint_{t, x}\left|\tau_{h} J_{1}-J_{1}\right|^{2}+2 \iint_{t, x}\left|\tau_{h} J_{2}-J_{2}\right|^{2} \\
& \leq C\|\phi\|_{W^{1, \infty}\left(\mathbb{R}^{3}\right)}^{2} N_{T}(u)^{2}\left[\frac{\exp \left(2 \lambda \epsilon^{2}\right)\left(\epsilon^{2 \gamma}+1\right)|h|}{\epsilon^{3}}+\left(\epsilon^{2}+\delta_{\gamma} \epsilon^{2 \gamma}\right)\right],
\end{aligned}
$$

for any $\epsilon>0$. Hence, (4.7) for the case of $i=1$ follows by taking $\epsilon=|h|^{1 / 5}$ if $\gamma=0$, and $\epsilon=|h|^{1 /(3+2 \gamma)}$ if $0<\gamma \leq 1$ for $|h| \leq 1$. The same argument leads to (4.7) when $i=2$.

Finally, we consider the case when $i=3$ in (4.7) whose proof needs some modification on the above proof for the case when $i=1$. In fact, similar to (4.10), (4.11) and (4.12), the velocity averaged function (4.3) of $S_{3}^{Q}$ can be rewritten as

$$
\int_{\mathbb{R}^{3}} S_{3}^{Q}(t, x, \xi) \phi(\xi) d \xi=\widetilde{J}_{1}+\widetilde{J}_{2}
$$

where

$$
\begin{aligned}
\widetilde{J}_{1} & =\iint_{\xi, \xi_{*}} \sqrt{\mathbf{M}(\xi)} u(t, x, \xi) \sqrt{\mathbf{M}\left(\xi_{*}\right)} u\left(t, x, \xi_{*}\right) \iint_{\eta, \eta_{*}} Z\left(\eta, \eta_{*}\right) \psi_{\epsilon}(\xi-\eta) \psi_{\epsilon}\left(\xi_{*}-\eta_{*}\right) \\
& =\iint_{\eta, \eta_{*}} Z\left(\eta, \eta_{*}\right) \rho_{\psi_{\epsilon}(-\eta \eta)}(\sqrt{\mathbf{M}} u)(t, x) \rho_{\psi_{\epsilon}\left(\cdot-\eta_{*}\right)}(\sqrt{\mathbf{M}} u)(t, x)
\end{aligned}
$$

and

$$
\widetilde{J}_{2}=\iint_{\xi, \xi_{*}} \sqrt{\mathbf{M}(\xi)} u(t, x, \xi) \sqrt{\mathbf{M}\left(\xi_{*}\right)} u\left(t, x, \xi_{*}\right) \iint_{\eta, \eta_{*}}\left[Z\left(\xi, \xi_{*}\right)-Z\left(\eta, \eta_{*}\right)\right] \psi_{\epsilon}(\xi-\eta) \psi_{\epsilon}\left(\xi_{*}-\eta_{*}\right) .
$$


Notice that

$$
\begin{aligned}
& \tau_{h}\left[\rho_{\psi_{\epsilon}(\cdot-\eta)}(\sqrt{\mathbf{M}} u) \rho_{\psi_{\epsilon}\left(\cdot-\eta_{*}\right)}(\sqrt{\mathbf{M}} u)\right]-\rho_{\psi_{\epsilon}(\cdot-\eta)}(\sqrt{\mathbf{M}} u) \rho_{\psi_{\epsilon}\left(\cdot-\eta_{*}\right)}(\sqrt{\mathbf{M}} u) \\
& =\tau_{h}\left[\rho_{\psi_{\epsilon}(\cdot-\eta)}(\sqrt{\mathbf{M}} u)\right]\left(\tau_{h}\left[\rho_{\psi_{\epsilon}\left(\cdot-\eta_{*}\right)}(\sqrt{\mathbf{M}} u)\right]-\rho_{\psi_{\epsilon}\left(\cdot-\eta_{*}\right)}(\sqrt{\mathbf{M}} u)\right) \\
& \quad+\left(\tau_{h}\left[\rho_{\psi_{\epsilon}(\cdot-\eta)}(\sqrt{\mathbf{M}} u)\right]-\rho_{\psi_{\epsilon}(\cdot-\eta)}(\sqrt{\mathbf{M}} u)\right) \rho_{\psi_{\epsilon}\left(\cdot-\eta_{*}\right)}(\sqrt{\mathbf{M}} u)
\end{aligned}
$$

and

$$
\iint_{\eta, \xi} \sqrt{\mathbf{M}(\xi)}|u(t, x+h, \xi)| \psi_{\epsilon}(\xi-\eta) \leq\|u\|_{L^{\infty}\left([0, T] \times \mathbb{R}_{x}^{3} \times \mathbb{R}_{\xi}^{3}\right)} \iint_{\eta, \xi} \sqrt{\mathbf{M}(\xi)} \psi_{\epsilon}(\xi-\eta) .
$$

Thus, $\iint\left|\tau_{h} \widetilde{J}_{1}-\widetilde{J}_{1}\right|^{2} d t d x$ can be estimated in a way similar to the first two cases. On the other hand, the estimate on $\iint\left|\tau_{h} \widetilde{J}_{2}-\widetilde{J}_{2}\right|^{2} d t d x$ follows by noticing that

$$
\begin{gathered}
\left|\tau_{h}\left[\sqrt{\mathbf{M}(\xi)} u(t, x, \xi) \sqrt{\mathbf{M}\left(\xi_{*}\right)} u\left(t, x, \xi_{*}\right)\right]-\sqrt{\mathbf{M}(\xi)} u(t, x, \xi) \sqrt{\mathbf{M}\left(\xi_{*}\right)} u\left(t, x, \xi_{*}\right)\right| \\
\leq\|u\|_{L^{\infty}\left([0, T] \times \mathbb{R}_{x}^{3} \times \mathbb{R}_{\xi}^{3}\right)} \sqrt{\mathbf{M}(\xi)} \sqrt{\mathbf{M}\left(\xi_{*}\right)}\left(\left|u\left(t, x+h, \xi_{*}\right)\right|+\left|u\left(t, x, \xi_{*}\right)\right|\right) .
\end{gathered}
$$

This completes the proof of the lemma.

Lemma 4.4. Let $\alpha_{0}(\gamma)$ be defined in (1.6). For each $i=1,2,3$ and for any $0<\alpha<\alpha_{0}(\gamma)$, we have

$$
S_{i}^{Q} \in L^{2}\left([0, T] \times B_{R_{\xi}} ; H^{\alpha}\left(B_{R_{x}}\right)\right) .
$$

Furthermore,

$$
\left\|S_{i}^{Q}\right\|_{L^{2}\left([0, T] \times B_{R_{\xi}} ; H^{\alpha}\left(B_{R_{x}}\right)\right)} \leq \frac{C K_{T}(u)\langle R\rangle^{3 / 2}}{\sqrt{\alpha_{0}(\gamma)-\alpha}},
$$

where $K_{T}(u)$ is given by (4.8).

Proof. Let $0<|h| \leq 1$. Take the mollifier $\psi_{\delta}(\cdot)$ with $\delta>0$ to be determined later. Write $S_{i}^{Q}$ as

$$
S_{i}^{Q}=\left(S_{i}^{Q}-\psi_{\delta} *_{\xi} S_{i}^{Q}\right)+\psi_{\delta} *_{\xi} S_{i}^{Q}
$$

Then

$$
\begin{aligned}
& \iiint_{>\mathbb{R}_{x}^{3} \times B_{R_{\xi}}} d t d x d \xi\left|\tau_{h} S_{i}^{Q}-S_{i}^{Q}\right|^{2} \\
& \leq 4 \iiint_{[0, T] \times \mathbb{R}_{x}^{3} \times B_{R_{\xi}}} d t d x d \xi\left|S_{i}^{Q}-\psi_{\delta} *_{\xi} S_{i}^{Q}\right|^{2}+2 \iint_{B_{R_{\xi}}} d \xi \iint_{[0, T] \times \mathbb{R}_{x}^{3}} d t d x\left|\tau_{h}\left(\psi_{\delta} *_{\xi} S_{i}^{Q}\right)-\psi_{\delta} *_{\xi} S_{i}^{Q}\right|^{2} .
\end{aligned}
$$

It follows from Lemmas $2.6,4.1$ and 4.3 that

$$
\begin{aligned}
& \iiint_{[0, T] \times \mathbb{R}_{x}^{3} \times B_{R_{\xi}}} d t d x d \xi\left|\tau_{h} S_{i}^{Q}-S_{i}^{Q}\right|^{2} \leq C\left\|S_{i}^{Q}\right\|_{L^{2}\left([0, T] \times \mathbb{R}_{x}^{3} ; \dot{H}^{1}\left(\mathbb{R}_{\xi}^{3}\right)\right)}^{2} \delta^{2} \\
& +C_{\psi} K_{T}(u)^{2} P_{\gamma}(h) \int_{B_{R_{\xi}}}\left\|\psi_{\delta}(\xi-\cdot)\right\|_{W^{1, \infty}\left(\mathbb{R}^{3}\right)} d \xi \leq C_{\psi} K_{T}(u)^{2}\langle R\rangle^{3}\left(\delta^{2}+\frac{P_{\gamma}(h)}{\delta^{8}}\right),
\end{aligned}
$$


where $P_{\gamma}(h)$ is defined by (4.9). We choose $\delta=|h|^{1 / 25}$ if $\gamma=0$, and $\delta=|h|^{\gamma / 5(3+2 \gamma)}$ if $0<\gamma \leq 1$ to have

$$
\iiint_{[0, T] \times \mathbb{R}_{x}^{3} \times B_{R_{\xi}}} d t d x d \xi\left|\tau_{h} S_{i}^{Q}-S_{i}^{Q}\right|^{2} \leq C_{\psi} K_{T}(u)^{2}\langle R\rangle^{3}|h|^{2 \alpha_{0}(\gamma)} .
$$

Then, for any fixed $\alpha \in\left(0, \alpha_{0}(\gamma)\right)$, we have

$$
\begin{aligned}
\iiint \int_{[0, T] \times B_{R_{x}} \times B_{R_{h}} \times B_{R_{\xi}}} \frac{\left|\tau_{h} S_{i}^{Q}-S_{i}^{Q}\right|^{2}}{|h|^{3+2 \alpha}} & \leq \int_{|h| \leq 1} \frac{C_{\psi} K_{T}(u)^{2}\langle R\rangle^{3}}{|h|^{3+2\left[\alpha-\alpha_{0}(\gamma)\right]}}+\int_{|h| \leq R}\left\|S_{i}^{Q}\right\|_{L^{2}\left([0, T] \times \mathbb{R}_{x}^{3} \times \mathbb{R}_{\xi}^{3}\right)} \\
& \leq \frac{C_{\psi} K_{T}(u)^{2}\langle R\rangle^{3}}{\alpha_{0}(\gamma)-\alpha}+C N_{T}(u)^{2}\langle R\rangle^{3} \leq \frac{C_{\psi} K_{T}(u)^{2}\langle R\rangle^{3}}{\alpha_{0}(\gamma)-\alpha} .
\end{aligned}
$$

This completes the proof of the lemma.

Corollary 4.1. Let $0<\alpha<\alpha_{0}(\gamma)$ with $\alpha_{0}(\gamma)$ defined in (1.6). For each $i=1,2,3$, we have

$$
\begin{gathered}
S_{i}^{Q}, Q^{+}(f, f) \in L_{\text {loc }}^{2}\left(\mathbb{R}_{+} ; H_{l o c}^{\alpha}\left(\mathbb{R}_{x}^{3} \times \mathbb{R}_{\xi}^{3}\right)\right) \\
0 \leq Q^{+}(f, f) \in L^{\infty}\left([0, T] \times \mathbb{R}_{x}^{3} \times \mathbb{R}_{\xi}^{3}\right)
\end{gathered}
$$

We are now ready to complete the proof for Theorem 1.1.

Proof of Theorem 1.1. By combining all estimates obtained above, we have (i) the mild form of the solution:

$$
f(t, x, \xi)=f_{0}(x-\xi t, \xi) \Gamma_{1}(t, x, \xi)+\Gamma_{2}(t, x, \xi),
$$

where

$$
\begin{aligned}
& \Gamma_{1}(t, x, \xi)=\exp \left(-\int_{0}^{t} L f(\theta, x-\xi \theta, \xi) d \theta\right) \\
& \Gamma_{2}(t, x, \xi)=\Gamma_{1}(t, x, \xi) \int_{0}^{t} Q^{+}(f, f)(s, x-\xi s, \xi) \exp \left(\int_{0}^{s} L f(\theta, x-\xi \theta, \xi) d \theta\right) d s .
\end{aligned}
$$

\section{(ii) The regularization estimates:}

$$
\begin{aligned}
& 0 \leq L f \in L^{2}\left([0, T] ; H_{l o c}^{1 / 2}\left(\mathbb{R}_{x}^{3} \times \mathbb{R}_{\xi}^{3}\right)\right) \cap L^{\infty}\left([0, T] \times \mathbb{R}_{x}^{3} ; L_{l o c}^{\infty}\left(\mathbb{R}_{\xi}^{3}\right)\right), \\
& 0 \leq Q^{+}(f, f) \in L^{2}\left([0, T] ; H_{l o c}^{\alpha}\left(\mathbb{R}_{x}^{3} \times \mathbb{R}_{\xi}^{3}\right)\right) \cap L^{\infty}\left([0, T] \times \mathbb{R}_{x}^{3} \times \mathbb{R}_{\xi}^{3}\right),
\end{aligned}
$$

for any $0<\alpha<\alpha_{0}(\gamma)$ with $\alpha_{0}(\gamma)$ defined by (1.6).

As in [8], the above estimates are sufficient to give (1.5)-(1.7) by straightforward calculation so that we omit the details. As for the perturbation $u$, since

$$
u(t, x, \xi)=u_{0}(x-\xi t, \xi) \Gamma_{1}(t, x, \xi)+\left[\sqrt{\mathbf{M}} \Gamma_{1}(t, x, \xi)+\frac{1}{\sqrt{\mathbf{M}}} \Gamma_{2}(t, x, \xi)-\sqrt{\mathbf{M}}\right],
$$

(1.8)-(1.9) follow from the estimation on $f$. This completes the proof of Theorem 1.1. 
Remark 4.1. Here we claim that the nonnegative functions $\Gamma_{1}(t, x, \xi)$ and hence $\widetilde{\Gamma}_{1}(t, x, \xi)$ decay exponentially for large $t$ uniformly in $x, \xi$, provided that the $L^{\infty}$-norm of $u$ over $\mathbb{R}_{+} \times$ $\mathbb{R}^{3} \times \mathbb{R}^{3}$ is small enough. This means that the singular part vanishes with an exponential rate. In fact, notice that

$$
\int_{0}^{t} L f(\theta, x-\xi \theta, \xi) d \theta=\nu(\xi) t+\int_{0}^{t} A *_{\xi}(\sqrt{\mathbf{M}} u)(\theta, x-\xi \theta, \xi) d \theta,
$$

where the collision frequency $\nu(\xi):=A *_{\xi} \mathbf{M}$ satisfies the condition, cf. [15, 25] that there is a positive constant $\nu_{0}$ such that

$$
\nu_{0}(1+|\xi|)^{\gamma} \leq \nu(\xi) \leq \frac{1}{\nu_{0}}(1+|\xi|)^{\gamma}
$$

Furthermore, one can see

$$
\left|A *_{\xi}(\sqrt{\mathbf{M}} u)\right| \leq C\|u\|_{L^{\infty}\left(\mathbb{R}_{+} \times \mathbb{R}_{x}^{3} \times \mathbb{R}_{\xi}^{3}\right)} \nu(\xi) .
$$

Thus one has

$$
0 \leq \Gamma_{1}(t, x, \xi) \leq \exp \left(-\nu(\xi)\left(1-C\|u\|_{L^{\infty}\left(\mathbb{R}_{+} \times \mathbb{R}_{x}^{3} \times \mathbb{R}_{\xi}^{3}\right)}\right) t\right)
$$

for any $t \geq 0, x \in \mathbb{R}^{3}, \xi \in \mathbb{R}^{3}$. Therefore, the aforementioned claim follows.

Acknowledgment: The research of Tong Yang was supported by the RGC Competitive Earmarked Research Grant of Hong Kong, CityU \#102606, and the Changjiang Scholar Program of Chinese Educational Ministry in Shanghai Jiao Tong University.

\section{References}

[1] R. Adams, Sobolev spaces, New York, Academic Press, 1985.

[2] R. Alexandre, L. Desvillettes, C. Villani, B. Wennberg, Entropy dissipation and long range interactions, Arch. Rat. Mech. Anal. 152 (2000), 327-355.

[3] R. Alexandre, M. ElSafadi, Littlewood-Paley decomposition and regularity issues in Boltzmann homogeneous equations. I. Non cutoff and Maxwell cases, Math. Meth. Models in Applied Sci. 15 (2005), 907-920.

[4] R. Alexandre, M. ElSafadi, Littlewood-Paley theory and regularity issues in Boltzmann homogeneous equations. II. Non cutoff case and non Maxwellian molecules, preprint (2007).

[5] R. Alexandre, Y. Morimoto, S. Ukai, C.-J. Xu, T. Yang, Uncertainty principle and kinetic equations, preprint.

[6] L. Bernis, L. Desvillettes, Propagation of singularities for classical solutions of the Vlasov-Poisson-Boltzmann equation, preprint (2007).

[7] F. Bouchut, L. Desvillettes, A proof of the smooothing properties of the positive part of Boltzmann's kernel, Rev. Mat. Iber. 14 (1998), 47-61. 
[8] L. Boudin, L. Desvillettes, On the singularities of the global small solutions of the full Boltzmann equation, Monatschefte für Mathematik 131 (2000), 91-108.

[9] L. Desvillettes, About the use of the Fourier transform for the Boltzmann equation, Riv. Mat. Univ. Parma 7 (2003), 1-99.

[10] L. Desvillettes, G. Furioli, E. Terraneo, Propagation of Gevrey regularity for solutions of Boltzmann equation for Maxwellian molecules, preprint (2007).

[11] L. Desvillettes, B. Wennberg, Smoothness of the solution of the spatially homogeneous Boltzmann equation without cutoff, Comm. Partial Differential Equations 29 (2004), 133-155.

[12] R. DiPerna, P.-L. Lions, On the Cauchy problem for the Boltzmann equation: Global existence and weak stability, Ann. Math. 130 (1989), 312-366.

[13] R.-J. Duan, On the Cauchy problem for the Boltzmann equation in the whole space: Global existence and uniform stability in $L_{\xi}^{2}\left(H_{x}^{N}\right)$, preprint (2007).

[14] F. Golse, P.-L. Lions, B. Perthame, R. Sentis, Regularity of the moments of the solution of a transport equation, J. Funct. Analysis 76 (1988), 110-125.

[15] H. Grad, Principles of the kinetic theory of gases, Flügge's Handbuch der Physik 12 (1958), 205-294.

[16] K. Hamdache, Existence in the large and asymptotic behaviour for the Boltzmann equation, Japan J. Appl. Math. 2 (1985), 1-15.

[17] R. Illner, M. Shinbrot, Global existence for a rare gas in an infinite vacuum, Comm. Math. Phys. 95 (1984), 117-126.

[18] K. Imai, T. Nishida, Global solutions to the initial value problem for the nonlinear Boltzmann equaion, Publ. RIMS Kyoto Univ. 12 (1976), 229-239.

[19] S. Kaniel, M. Shinbrot, The Boltzmann equation I: uniqueness and global existence, Comm. Math. Phys. 58 (1978), 65-84.

[20] P.-L. Lions, Compactness in Boltzmann's equation via Fourier integral operator and applications, Part I-II \& III, J. Math. Kyoto Univ. 34 (1994), 391-461 \& 539-584.

[21] X. Lu, A direct method for the regularity of the gain term in the Boltzmann equation, J. Math. Anal. Appl. 228 (1998), 409-435.

[22] Y. Morimoto, S. Ukai , C.-J. Xu, T. Yang, Regularity of solutions to the spatially homogeneous Boltzmann equation without angular cutoff, preprint.

[23] C. Mouhot, C. Villani, Regularity theory for the spatially homogeneous Boltzmann equation with cut-off, Arch. Ration. Mech. Anal. 173 (2004), 169-212.

[24] S. Ukai, On the existence of global solutions of mixed problem for non-linear Boltzmann equation, Proceedings of the Japan Academy 50 (1974), 179-184. 
[25] S. Ukai, Solutions of the Boltzmann equation. In: "Pattern and Waves-Qualitive Analysis of Nonlinear Differential Equations" (Mimura, M. and Nishida, T., eds.), Studies of Mathematics and Its Applications, Vol. 18, Kinokuniya-North-Holland, Tokyo, 1986, pp. 37-96.

[26] S. Ukai and T. Yang, The Boltzmann equation in the space $L^{2} \cap L_{\beta}^{\infty}$ : Global and time-periodic solutions, Analysis and Applications 4 (2006), 263-310.

[27] C. Villani, A review of mathematical topics in collisional kinetic theory. Handbook of mathematical fluid dynamics, Vol. I, 71-305, North-Holland, Amsterdam, 2002.

[28] B. Wennberg, Regularity in the Boltzmann equation and the Radon transform, Comm. Partial Differential Equations 19 (1994), 2057-2074. 